\title{
Case Study Examples of Interoperable Ethernet Communications Within Distribution, Transmission, and Wide-Area Control Systems
}

\author{
David Dolezilek, Schweitzer Engineering Laboratories, Inc.
}

\begin{abstract}
While special protection systems (SPSs) often shed load, recent sophisticated remedial action schemes (RASs) reduce or terminate generation output during an emergency condition. Under certain load conditions, generation newly added to previously balanced transmission grids creates system conditions that violate accepted reliability criteria.

At Southern California Edison (SCE), RAS systems are implemented to ensure reliable power system performance following outages on a transmission grid network. They include fast, automatic control actions to mitigate thermal overloads and system instability upon the loss of one or more transmission lines. With these automatic protection features, RAS systems are used in place of expensive alternative measures, which include reconductoring transmission lines, building new lines, and/or adding new transformers. Testing at SCE demonstrates the successful use of IEC 61850 GOOSE (Generic Object-Oriented Substation Event) messages over a distance up to 720 kilometers to collect analysis and arming data and transfer status and control indications. Complete detection, alarming, calculation, and remediation are completed in well under the 50-millisecond benchmark.

Using standardized IEC 61850 GOOSE methods avoids the customization required to implement individual local RAS communications systems, allows centralized coordination of arming, disarming, and system testing, and simplifies the coordination of system maintenance. Reliability improves with capabilities to monitor end-to-end grid parameters and quickly respond to abnormal conditions.

These methods of mitigation are intended to be used throughout the SCE area of operation as well as at all interties to neighboring utilities to facilitate dynamic load shedding/generation tripping and improved load management.

SPSs and RASs must be put in place to protect existing systems that are called upon to serve new generation and load, are intertied with weak systems, or have geographical characteristics that reduce stability. Once protected, the automatic load and generation control ensures stability while improving production and reliability. Once these are in place, wide-area monitoring and control are safely added to replace state estimation with real-time state measurement and management.
\end{abstract}

\section{INTRODUCTION}

Contemporary SPSs (special protection systems) and RAS (remedial action scheme) systems are deployed via a network of intelligent electronic devices (IEDs) that monitor field conditions and react to contingency control actions. Digital communication among these IEDs is similar to that for substation automation systems (SASs) and distribution automation (DA), except that it generally travels farther to perform wide-area control. Protection, control, and monitoring (PCM) IEDs often also serve as monitor and control devices for RAS systems. However, separate IED networks are often deployed for physical segregation to create resiliency and autonomy of the data paths and information processing. Information processing includes the results of specialized calculations within IEDs, data sharing among networked IEDs, and the processing of data to create power system information. These PCM or RAS networks and associated applications create the "operational technology" (OT) that automatically and manually generates, transmits, distributes, and consumes both energy and information about these processes. Information technology (IT) methods collect data and information from the OT network and distribute them to people and processes that use them for decision-making purposes.

The IT world is quickly moving to IP (Internet protocol) and other packet-based technologies because of convenience, flexibility, and multipurpose networking. OT networks have traditionally been data pipe-oriented communications that have dedicated bandwidth for each connection. Pipe-oriented communications often use nonroutable protocols, while packet-oriented communications use routable protocols. Dedicated bandwidth OT data pipe-oriented methods like time division multiplexing (TDM) are cautiously being replaced with packet-based communications, because the IT features cause detrimental behavior to OT applications. Careful attention to resiliency, deterministic behavior, and reliability has forced the industry to adapt IP and packet-based communications to work acceptably in the OT world. Specifically, many necessary changes to make Ethernet useful for electric utility OT have been made by IEEE and adopted by IEC.

OT versus IT methods and nonroutable versus routable protocols play an important role in the design and performance of RAS, SPS, SAS, and DA systems.

\section{RAS USING NONROUTABLE PROTOCOL}

The Blythe Energy Power Plant (BEPP), located in Blythe, California, was designed to incorporate a gas-fired, combinedcycle configuration consisting of two $175 \mathrm{MW}$ combustion turbine generators and one $170 \mathrm{MW}$ steam turbine generator. The $520 \mathrm{MW}$ electrical output is connected to the regional transmission grid via the existing transmission system 
managed by the Western Area Power Administration (Western). Reference [1] describes the successful use of the MIRRORED BITS ${ }^{\circledR}$ communications protocol, which is a serial peer-to-peer communications technology that exchanges the status of Boolean and analog data, encoded in a digital message, from one device to another. This inexpensive, highly secure technology is used in numerous protection, control, automation, and monitoring applications within BEPP, SCE (Southern California Edison), and around the world. It is one of the most popular nonroutable, data pipe-oriented methods within electric utility OT.

Impacts to existing power systems, typically caused by adding new generation to the established transmission grid, often include overloaded transmission lines, transformers, circuit breakers, and other system components that may cause violations of accepted reliability criteria. The North American Electric Reliability Corporation (NERC), Western Electricity Coordinating Council (WECC), and local reliability requirements determine the criteria for California installations, including the Blythe substation. To mitigate potential reliability problems, Western deployed a RAS system prior to connecting BEPP to the transmission grid. This RAS system provides generation reduction capabilities during transmission line overload conditions. Recognizing the increasing importance of having reliable RAS systems, Western chose to implement this system in a dual-primary design so that no single device or connection would be a single point of failure. Reference [1] discusses the design and implementation of these RAS systems using protective relays, communications processors, digital I/O modules, and an I/O processor.

\section{COMPARING CENTRALIZED RAS SYSTEMS USING MIRRORED BITS COMMUNICATIONS AND IEC 61850 GOOSE}

SCE documented a case study comparing the performance of multiple communications technologies and architectures available via protection and automation IEDs for use in monitoring and controlling a RAS. The discussion includes the design description and implementation issues of several popular and standardized technologies available today to perform high-speed digital data communications among IEDs. Reference [2] describes analysis of the serial and Ethernet methods for transferring MIRRORED BITS communications and IEC 61850 GOOSE (Generic Object-Oriented Substation Event) messages.

\section{A. The SCE Reasons for a New RAS Approach}

SCE deploys local RAS systems throughout their transmission operating area, including 1,183 miles of $500 \mathrm{kV}$ lines, 1,181 miles of $230 \mathrm{kV}$ lines, and 350 miles of $115 \mathrm{kV}$ lines. Supporting these main transmission corridors are several independent localized RAS systems, with more systems under development and the potential to add a multitude of new systems based on recent generator queue studies. Perhaps most important is the anticipation of creating RAS systems that cover very large areas. These newer systems will need to not only accept many messages simultaneously from many remote locations but also process each message and then the associated RAS logic.

System reliability is expected to improve with capabilities to monitor end-to-end grid parameters and quickly respond to abnormal conditions. The mitigation area will expand from a few local choices to all nodes included within the SCE system, including dynamic load shedding/generation tripping and improved load restoration management. A new centralized RAS (CRAS) system will mirror the success of the localized systems to a wide-area RAS that covers the SCE large service territory.

\section{B. Design for Reliability and Decision Analysis Predict System Availability and Value}

Functionally, IEDs networked together into a SAS provide operational SCADA (supervisory control and data acquisition) data, engineering and analysis access, and high-speed interdevice data exchange. Reference [3] identifies the major selection and design criteria of network functionality, components, and topology. It examines and compares serial and Ethernet architectures for an example substation using the following criteria: reliability; cost of equipment and commissioning; safety; ease and cost to design, implement, maintain, and expand; effective data transfer rates; and performance of high-speed control signals.

IEC 61850-3 Section 4 also describes that each system shall be designed as a fail-safe design such that:

"...There shall be no single failure mode that causes the SAS to initiate an undesired control action, such as tripping or closing a circuit breaker. In addition, SAS failures shall not disable any available local metering and local control functions at the substation" [3].

IEC 61850-3 Section 4 describes the following reliability measures for design comparison:

- Reliability measured as MTBF (mean time between failures).

- Device availability measured as percent availability.

- System availability measured as percent availability.

- Device maintainability measured as MTTR (mean time to repair).

- System maintainability measured as MTTR [3].

\section{SCE RAS MESSAGE PERformanCE ANALysis}

\section{A. Speed and Control Timing}

SCE established a benchmark of 50 milliseconds to detect a change, evaluate contingencies, and respond with RAS control actions in three-IED scenarios [2]. This time includes remotely detecting an abnormal condition, transmitting an alarm 460 miles over a WAN (wide-area network) to the centralized RAS controller, determining the proper actions, and then transmitting these actions 460 miles over a WAN to the appropriate remote RAS IEDs where the control actions are implemented. 


\section{B. Test Description}

The test involves three IEDs communicating to each other. IED1, the monitor IED, monitors line conditions and, when appropriate after a line-open condition is detected, sends a status message to IED2, the central logic processor IED. The status of the RAS, armed or disarmed, is resident in IED2, as is the logic to determine when to send a mitigation signal. The line-open condition is simulated by energizing an input contact on IED1. Upon receipt of the status message from IED1, IED2 extracts its content and, if the RAS is armed, performs a calculation to determine if remedial action is necessary. If IED2 decides to take action, IED2 sends a mitigation command message to IED3, the mitigation IED. When IED3 receives the mitigation command message from IED2, IED3 energizes a trip output contact. This output contact is hard-wired to an input on IED1. In this way, the total time duration is measured between detection of a lineopen condition as a contact input on IED1 and the eventual trip output of IED3 detected as a second contact input on IED1. The time duration is measured with a separate instrument and verified with internal Sequential Events Recorder (SER) reports.

SCE staged the test with IEDs from two different vendors and tested three different protocols. IEDs from one of the vendors, referenced as Vendor A, were tested with two different protocols enabled. These tests were completed on a LAN (local-area network with all IEDs directly connected peer to peer or via a local Ethernet switch) and across a WAN connection via a local Ethernet switch and Ethernet router.

GOOSE protocol messages are published to a multicast group address and are not routable over a WAN. In order to simulate WAN timing for the tests, SCE actually created a long LAN connection over the physical WAN connection, via the SONET (synchronous optical network) system between Los Angeles and Bakersfield, California. This is actually a data pipe that transfers data packets over a dedicated bandwidth channel. SCE recognized that, unlike nonroutable MIRRORED BITS communications, the packet-oriented GOOSE protocol installations required logical LAN connections between all RAS locations. This raised severe security concerns that needed to be addressed separately.

\section{Test Results}

Table I shows the timing results of the tests performed. LAN and WAN peer-to-peer times were calculated based on SER records in the IEDs. All roundtrip time results for the three-IED test scenario were also measured externally using a scope.
TABLE I

IED TIMING RESULTS FOR RAS SYSTEM PROTOCOL TESTS USING THE THREE-IED RAS SCENARIO

\begin{tabular}{c|c}
\hline Test Case & $\begin{array}{c}\text { IED } \\
\text { Peer-to-Peer } \\
\text { Time }\end{array}$ \\
\hline Vendor A GOOSE protocol via Ethernet LAN & $4 \mathrm{~ms}$ \\
\hline Vendor A GOOSE protocol via Ethernet to WAN & $9 \mathrm{~ms}$ \\
\hline Vendor A MIRRORED BITS via serial LAN & $4 \mathrm{~ms}$ \\
\hline Vendor A MIRRORED BITS via serial to WAN & $9.2 \mathrm{~ms}$ \\
\hline
\end{tabular}

\section{System Reliability Analysis}

Using fault tree analysis, SCE calculated the reliability of each system type to compare relative dependability and uptime. Table II lists the calculated expected downtime, which is a measure of unreliability due to the unavailability of the RAS system. Each time a system becomes unavailable, it also requires a substantial maintenance effort to return it to service.

TABLE II

RELIABILITY ANALYSIS OF COMMUNICATIONS SYSTEM ARCHITECTURES Via FAULT TREE ANALYSIS

\begin{tabular}{c|c|c}
\hline Test Case & Availability & $\begin{array}{c}\text { Predicted Average } \\
\text { Annual Out-of- } \\
\text { Service Minutes }\end{array}$ \\
\hline $\begin{array}{c}\text { Vendor A GOOSE protocol } \\
\text { via Ethernet LAN }\end{array}$ & $99.982 \%$ & 97 \\
\hline $\begin{array}{c}\text { Vendor A GOOSE protocol } \\
\text { via Ethernet to WAN }\end{array}$ & $99.962 \%$ & 0 \\
\hline $\begin{array}{c}\text { Vendor A MIRRORED BITS } \\
\text { via serial LAN }\end{array}$ & $100 \%$ & 37 \\
\hline $\begin{array}{c}\text { Vendor A MIRRORED BITS } \\
\text { via serial to WAN }\end{array}$ & $99.993 \%$ & 00 \\
\hline
\end{tabular}

The use of digital RAS communications realizes significant system benefits over traditional methods of using multiple copper terminations to measure field contact status, regardless of the protocol(s) or communications media. The reduced number of field terminations, associated wiring, labor, and maintenance due to the reuse of data detected by a single IED, digitally communicated to integrated IEDs and other data clients, led SCE to determine the following:

- The nonroutable, data pipe-oriented MIRRORED BITS communications systems meet all acceptance criteria of the CRAS system.

- The packet-oriented GOOSE protocol over Ethernet meets the performance acceptance criteria of the CRAS system.

Both GOOSE and MIRRORED BITS communications protocols are available to all vendors for purchase or license to be included in their products. 


\section{REAL-Time Diagnostics BeCOME ESSENTIAL TO SUCCESS}

During testing, SCE noticed an inability to verify correct operation of GOOSE messages on the Ethernet network unless the IEDs provided diagnostics. SCE found it essential that the IEDs provide such diagnostics to complement analysis available via network analyzers. Reference [4] illustrates diagnostics that provide necessary IED status and messaging status information directly from the in-service IED.

\section{A. GOOSE Message Performance and Quality Monitoring}

IEDs exchanging GOOSE messages automatically monitor the communications to determine message quality. Each device detects errors in received messages and the failure to receive expected messages from other devices and performs remediation immediately. The error codes indicating bad quality are summarized in Table III. If the IED detects any of these to be true, it sets the message quality to failure.

TABLE III

GOOSE MESSAGE ERROR CODES

\begin{tabular}{c|c}
\hline Message Statistics & Error Code \\
\hline $\begin{array}{c}\text { Configuration revision mismatch } \\
\text { between publisher and subscriber }\end{array}$ & CONF REV MISMA \\
\hline $\begin{array}{c}\text { Publisher indicates that } \\
\text { it needs commissioning }\end{array}$ & NEED COMMISSIO \\
\hline Publisher is in test center & TEST MODE \\
\hline $\begin{array}{c}\text { Received message is decoding } \\
\text { and reveals error }\end{array}$ & MSG CORRUPTED \\
\hline Message received out of sequence & OUT OF SEQUENC \\
\hline Message time to live expired & TTL EXPIRED \\
\hline
\end{tabular}

\section{B. Uniquely Identify Each Configuration Revision in the IED}

IEC 61850 describes the Substation Configuration Language (SCL) and configuration files that configure devices for IEC 61850 communications. The preferred method is to load a configuration file, rather than individual settings, into the IED. Loading the file directly into the IED has several advantages over the legacy method of sending settings. A very important advantage is the ability to identify what communications behavior the IED is configured for by retrieving the filename and configuration revision directly from the IED while it is in service. Then it is possible to crossreference the behavior of this IED and the behavior of other IEDs with the configuration files.

Further, by separating IEC 61850 configuration from other IED automation and protection settings via the SCL configuration file download, it is possible to be certain that no other settings were accidently modified or affected. This provides security by minimizing the impact to the system, minimizing the recommissioning after a change, and eliminating the risk of unintentionally affecting the other processes within the system.
IEDs that support a GOOSE report provide real-time status of incoming and outgoing GOOSE messages and their configuration. Each report includes message configuration and performance information for each GOOSE message being published and for those to which the IED has subscribed.

Fig. 1 illustrates a GOOSE report collected directly from an in-service IED named PAC_MASTER. This report provides essential configuration parameters and diagnostics.

The GOOSE transmit status documents the configuration of the IED from which the report was retrieved.

The suffix 01 of the reference name confirms that the SCL file active in the IED is Revision 01.

The multicast address, priority, VLAN, state number, sequence number, data set name, time to live, and error code are each displayed for each GOOSE message being published.

The GOOSE receive status documents the configuration of the IEDs and the associated GOOSE subscriptions configured to be received. Elements of the third subscription are highlighted to show configuration revision and error code.

The third subscribed GOOSE message is from an IED named PAC_SLAVE_B.

The suffix 01 of the reference name for PAC SLAVE B confirms that the SCL file active in the IED is Revision 01.

In this case, the message is not being received and so the time to live has expired.

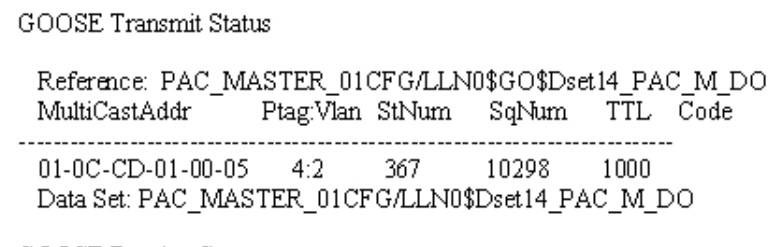

GOOSE Receive Status

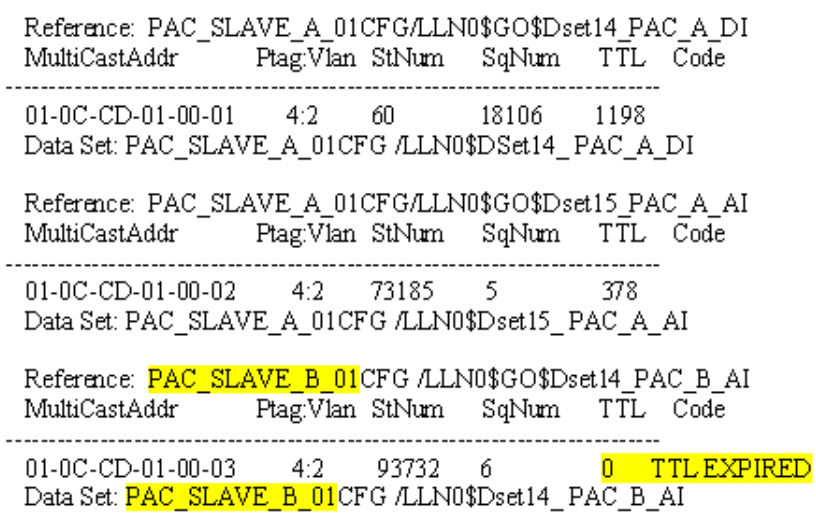

Fig. 1. PAC_MASTER GOOSE Report Showing Transmit and Receive Configuration and Status 


\section{Calculate and Visualize GOOSE Message Reliability and Channel Availability}

Once calculated and recorded as a time-stamped SER, each GOOSE message quality status is used to calculate reliability and availability. Message quality indicates failure when a message is corrupted or not received within the time to live. The observation of failures indicates the reliability of individual GOOSE messages. If the message quality failure is intermittent, the duration of the failures is calculated as the difference between time stamps. The aggregate of failure duration over a given amount of time determines the channel availability. Fig. 2 illustrates the use of the GOOSE quality status to alert users of a failed GOOSE subscription via the front-panel HMI (human-machine interface) to aid diagnostics and troubleshooting. In this case, a GOOSE message with analog inputs (AI) from the IED labeled $C$ has failed, while the one labeled $B$ is normal. It is also possible to use the status to trigger text and email messages to alert remote technicians.

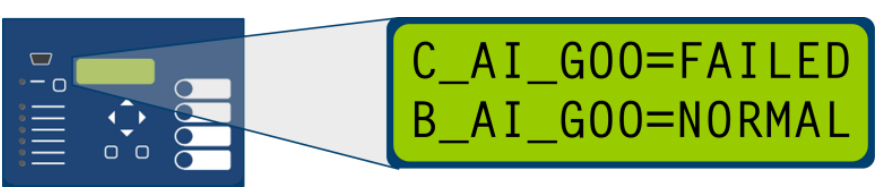

Fig. 2. PAC HMI View of GOOSE Message Quality Display Point

\section{IMPROVING THE STATE OF THE ART WITH SYNCHROPHASORS}

To date, synchronized phasor measurements have been used mainly for power system model validation, post-event analysis, real-time display, and other similar activities. However, synchrophasors have a greater potential than monitoring and visualization. Synchrophasors will increasingly contribute to the reliable and economical operation of power systems as real-time control and protection schemes become broadly used. Synchronous phasor measurements are now available in relays and meters; however, a practical means of processing the data in real time has been lacking.

Reference [5] describes the synchrophasor vector processor (SVP) and several practical applications, including automated diagnostics, RAS, direct state measurement, and stability assessment. This real-time synchrophasor processor device further improves RAS and SPS systems by performing vector mathematics in real time.

\section{A. The SVP}

The purpose of the SVP is to collect synchronous phasor measurements (SPMs), collect logical inputs, perform vector and scalar calculations, make decisions, produce outputs, and report data. A simple task for an SVP might be collecting SPMs from two ends of a transmission line, comparing the voltage angles, and issuing a warning to an operator if a threshold has been exceeded. A more complicated example might be distributed SVPs performing localized substate measurement and forwarding results to a higher level to build the entire state vector in real time, without the nonlinear and time-consuming steps of state estimation.

\section{B. Traditional RAS System Implementation}

Fig. 3 shows the timing diagram of a traditional digital communications RAS system, including the relay detection time and relay assertion output time. The system consists of approximately 73 individual pieces of equipment, including $\mathrm{I} / \mathrm{O}$ modules and logic processors.

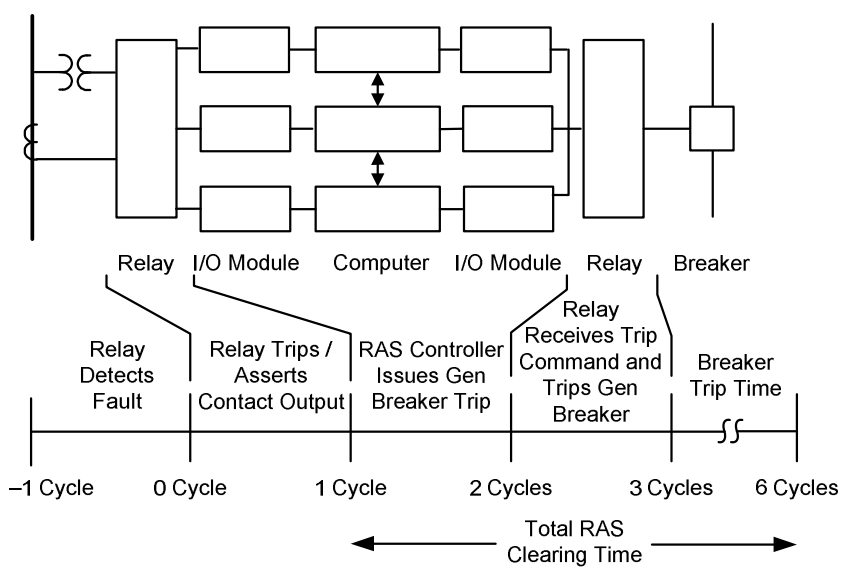

Fig. 3. RAS Clearing Time Budget

\section{SVP Implementation}

For the SVP RAS implementation, the relays forward synchrophasor data, and the SVP determines if there is a loss of load, overpower, etc. The net result is that implementing an SVP solution and using high-speed communications tripping can save three-quarters of a cycle.

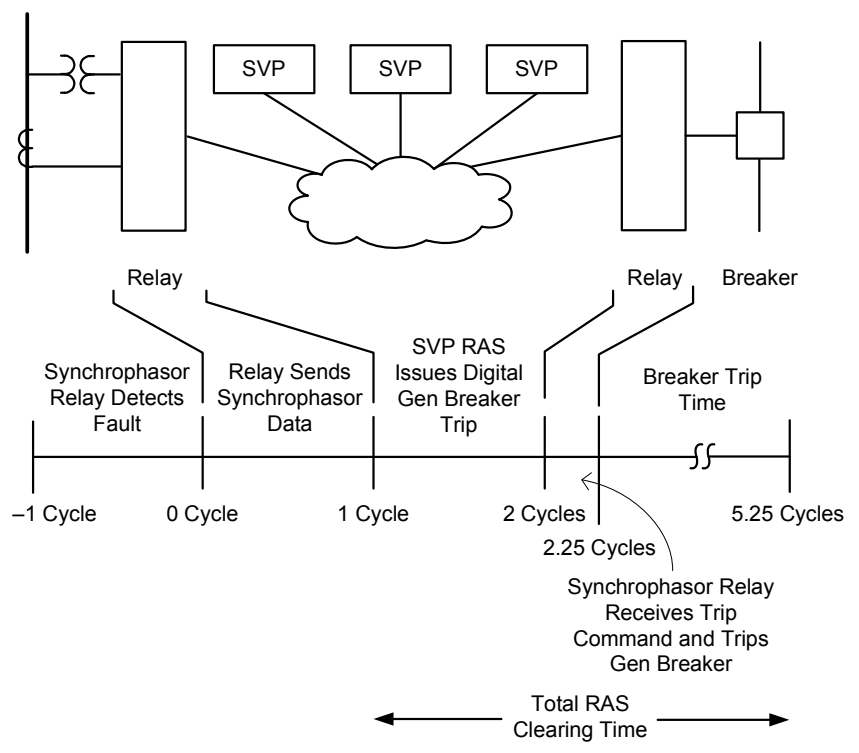

Fig. 4. SVP RAS Clearing Time 


\section{CONCLUSIONS}

Fast and reliable RAS and SPS technologies maximize the efficiency of new and in-service generation, transmission, and distribution assets.

- Adding new generation to the transmission grid can impact the existing power system by potentially violating reliability criteria.

- Impacts to existing power systems, typically caused by adding new generation to the established transmission grid, often include overloaded transmission lines, transformers, circuit breakers, and other system components that may cause violations of accepted reliability criteria. RAS schemes are designed to rapidly acquire power system measurements and manage generation and load to prevent violations and provide stability.

- The use of digital communications for RAS data acquisition and control realizes significant system benefits over traditional methods of using multiple copper terminations to measure field contact status, regardless of the protocol(s) or communications media. The number of field terminations, associated wiring, labor, and maintenance are reduced because of the reuse of data communicated digitally.

- IEC 61850-3 Section 4 summarizes design practices and reliability measures useful to maximize system reliability and availability.

- GOOSE reports provide quick troubleshooting diagnostics by documenting configuration and status of incoming and outgoing GOOSE messages.

- Now that SPMs are broadly available from protective relays and meters, it is time to put them to work to improve our power systems. The SVP makes real-time applications practical.

- Direct state measurement is now practical because of the widespread availability of SPMs. The SVP plays a role in direct state measurement and can actually reduce the amount of information communicated to the master station.

\section{REFERENCES}

[1] M. Agudo, D. Fox, D. Dolezilek, and R. Jenkins, "Case Study: Integrate Substation IEDs to Provide Reliable, Independent Dual-Primary Remedial Action Schemes," proceedings of the 5th Annual Power Systems Conference, Clemson, SC, March 2006.

[2] M. Gugerty, R. Jenkins, and D. Dolezilek, "Case Study Comparison of Serial and Ethernet Digital Communications Technologies for Transfer of Relay Quantities," proceedings of the 33rd Annual Western Protective Relay Conference, Spokane, WA, October 2006.

[3] G. W. Scheer and D. Dolezilek, "Selecting, Designing, and Installing Modern Ethernet or Serial Digital Data Networks in Electrical Substations," proceedings of the 9th Annual Western Power Delivery Automation Conference, Spokane, WA, April 2007.

[4] R. Jenkins and D. Dolezilek, "Case Study: Using IEC 61850 Methods for RTU Replacement and Distributed Automation," proceedings of the 10th Annual Western Power Delivery Automation Conference, Spokane, WA, April 2008.

[5] E. O. Schweitzer, III and D. Whitehead, "Real-Time Power System Control Using Synchrophasors," proceedings of the 34th Annual Western Protective Relay Conference, Spokane, WA, October 2007.

\section{BIOGRAPHY}

David J. Dolezilek is the technology director of Schweitzer Engineering Laboratories, Inc. $\mathrm{He}$ is an electrical engineer, BSEE Montana State University, with experience in electric power protection, integration, automation, communications, control, SCADA, and EMS. He has authored numerous technical papers and continues to research innovative technology affecting our industry. Dolezilek is a patented inventor and participates in numerous working groups and technical committees. He is a member of IEEE, the IEEE Reliability Society, CIGRE working groups, and two International Electrotechnical Commission (IEC) Technical Committees tasked with global standardization and security of communications networks and systems in substations. 